\title{
Spectral engineering and tunable thermoelectric behavior in a quasiperiodic ladder network
}

\author{
Amrita Mukherjee* \\ Department of Physics, University of Kalyani, Kalyani, West Bengal-741 235, India
}

\author{
Atanu Nandy ${ }^{\dagger}$ \\ Department of Physics, Kulti College, Kulti, Paschim Bardhaman, West Bengal-713 343, India
}

\begin{abstract}
Double-stranded quasiperiodic copper mean arrangement has been studied in respect of their electronic property and thermoelectric signature. The two-arm network is demonstrated by a tightbinding Hamiltonian. The eigenspectrum of such aperiodic mesh that does not convey translational invariance, is significantly dependent on the parameters of the Hamiltonian. It is observed that specific correlation between the parameters obtained from the commutation relation between the on-site energy and overlap integral matrices can eventually modify the spectral nature and generate absolutely continuous energy spectrum. This part is populated by atypical extended states that has a large localization length substantiated by the flow of the hopping integral under successive real space renormalization group method steps. This sounds delocalization of single particle energy states in such non-translationally invariant networks. Further this can be engineered at will by selective choice of the relative strengths of the parameters. This precise correlation has a crucial impact on the thermoelectric behavior. Anomalous nature of thermoelectric coefficient may inspire the experimentalists to frame tunable thermo-devices. Specific correlations can help us to tune the continuous band and determine the band position at will.
\end{abstract}

PACS numbers: 71.30.+h, 72.15.Rn, 03.75.-b

\section{INTRODUCTION}

The unique concept of localization of excitation [1-3] in disordered media has recently covered diverse area of physics beyond its original electronic proposition. Starting from acoustics [4] to Bose Einstein condensates [5] as well as in the context of plasmonic $[6,7]$, phononic [8], polaronic excitations [9] the central issue of localization is very pertinent. The key idea has gained renewed interest due to the evident potential for the realization of localization of optical waves in random media. Recent path-breaking experimental observations of Photonic localization $[10,11]$ have added additional momentum in this realm. Not only that the localization of light using path-entangled photons [12] and tailoring of partially coherent light [13] have aroused extra inspiration from the realistic point of view.

Now several observations regarding the interesting variation of the classic case of the Anderson localization have drawn much attention over the past few years in the context of disordered systems. This was started from the concept of geometrically correlated disorder in the distribution of on-site energies, the so called random dimer model (RDM) [14]. Not only this, the disordered arrangement of overlap integrals [15] or quite recently the controlled engineering of absolutely continuous band structure in quasi one dimensional or quasiperiodic networks [16-21] - all those have offered significant physics

\footnotetext{
*Electronic address: amritaphy92@gmail.com

${ }^{\dagger}$ Electronic address: atanunandy1989@gmail.com
}

and prospects of designing novel devices in the era of advanced nanotechnology and lithography technique. Suitable correlation between the parameters of the Hamiltonian can lead to generate absolutely continuous bands populated by delocalized Bloch-like single particle eigenfunctions. Controlled engineering of band structure by correlated manipulation of parameters or even by tuning the external magnetic perturbation sometimes, may be useful for fabricating some photonic or thermoelectric devices.

In this communication, we address the possible analytical prescription of engineering the absolutely continuous bands populated by extended eigenfunctions in case of two-legged ladder network which is essentially made of some well-known quasiperiodic geometry. This system in fact does not have any translational invariance. The quasiperiodic arrangement has a finite extent in the transverse direction but possesses end-less axial span. Though problem of band tuning we have discussed here is in the context of quasiperiodic geometry, it is needless to mention that it is also equally valid for any disordered environment. Particularly we have focused on a deterministic quasiperiodic sequence because the exact analytical treatment is possible using the real space renormalization group method in the tight-biding formalism.

We concentrate on the ladder network arranged in a quasiperiodic Copper-mean (CM) [22] sequence. Though any arbitrary number of periodic stacking in the $y$ direction of the basic infinitely long two-legged ladder constituting a quasiperioidic mesh is in principle possible, we discuss the basic two-arm case only, the multichannel quasiperiodic mesh is just the extended version that contributes nothing additional spectral features but 
only contain rigorous mathematics. We discuss elaborately the detailed analysis related to band engineering and its effect on conductivity considering the two-legged CM ladder. This is illustrated in the Fig. 1.

Moreover, the methodology is easily extendable to randomly disordered system of arbitrary width. In case of random dimer model (RDM), the typical correlated clusters of atomic sites causes local resonances. Whereas here the spectral nature is solely dependent on the competition between the horizontal quasiperiodic ordering and the transverse periodicity (as the system grows vertically). This competition makes the scenario more interesting and challenging. So the deduction of resonance criteria or more specifically speaking, exact numerical correlation between the parameters for the occurrence of Bloch-like extended single particle states thus turns out to be quite non-trivial. The 'deterministic' growth pattern of this quasiperiodic sequence helps us to solve the problem in the tight-binding framework. Taking the advantage of this we demonstrate the technique of controlled engineering of electronic states in quasiperiodic ladder networks and we solidify our analysis by computing the exact numerical correlation, and justify it by evaluating the density of states using the Green's function technique. We will obtain the exact mathematical criteria for generation of transparent band and substantiate our discussion by computing the transport characteristics.

The strength of our analytical attempt lies in the fact that we can obtain an exact mapping of a two or multiarm quasiperiodic ladder network into a set of completely 'isolated' linear chains that describe the quantum mechanics of a class of pseudoparticles. Such an exact mapping has already been demonstrated in ladder-like geometries [16, 17, 23] modelling a DNA-like double chain or a quasi-two dimensional mesh with correlated disorder in the context of de-localization of single particle eigenstates.

In the next part, we study the thermoelectric transport property of these aperiodic ladder geometries in the tight-binding formalism. Suitable parametric correlation induced spectral nature can make the system transparent to the incoming electron for selective zone of Fermi energy and this selection can be controlled at will. In this work, we try to explore this correlation dependent transmission profile for studying the thermoelectric behavior of the system arranged in a quasiperiodic fashion.

The study of charge transport in molecules has potential applications in molecular electronics [24] and energyconversion devices $[25,26]$ such as electron transfer, shot noise, heat transport, negative differential resistance and gate controlled effects. Recently, topics on thermorelated transport such as local heating and thermal transport have emerged as new sub-field in molecular electronics. One of the significant aspect in the molecular tunnel junction is thermoelectricity. The Seebeck coefficient which is basically the slope of the transmission function in the vicinity of Fermi energy level, can carry more significant information than simple current-voltage characteristics. The study of thermoelectricity has its main importance in designing some thermorelated electronic and nano scale energy conversion devices. The experimental study of thermoelectric voltage over guanine molecules adsorbed on a graphite substrate reported by Poler et. al. [27] has opened novel perspectives in the quest for thermoelectric devices based on molecular electronics engineering. From the theoretical point of view, the extreme response of thermopower to finer details in the electronic structure allows us to extract considerable information regarding the relative position of Fermi level compared to molecular levels and also from the practical perspective, in order to optimize the thermoelectric figure of merit of a given molecular arrangement. Inspired by lots of experimental measurements of thermopower and comparison with the theoretical results for some organic molecular systems [28-30] here we try to figure out the flavor of correlation dependent tunable thermoelectric behavior for this model network within the tight-binding prescription. We find interesting results. For a multi-arm copper-mean

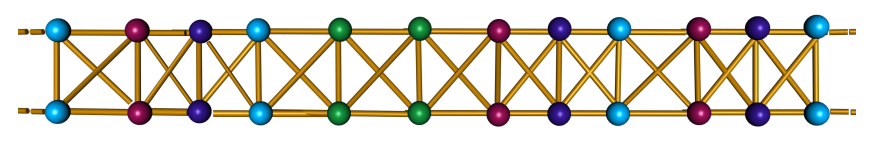

FIG. 1: (Color online) Schematic diagram of two-strand copper-mean ladder network. The different quantum dots are designated by different colors such as green dot $(\alpha$ site $)$, red $\operatorname{dot}(\beta$ site $)$, purple dot $(\gamma$ site) and cyan dot $(\delta$ site $)$ respectively. Two types of bonds 'long' and 'short' bonds are assigned with two different hopping integrals $t_{A}$ and $t_{B}$ respectively.

mesh, it is possible to give an exact analytical correlation between the parameters of the Hamiltonian. For twoarm case, there can be two such possibilities of different choices of correlation. For odd-strand network, there will be $N-1$ different choices of correlations, where $N$ is the number of strand. For all such cases, the overall spectrum loses its quasiperiodic identity. The inter-strand connection also plays a significant role in this context. The transport behavior is also tested by virtue of usual technique. This gives a strong support to the spectral nature. In the second part of our work, we find the correlation induced thermoelectric property. It shows an anomalous behavior with energy. This correlation dependence could make the system more appreciating in the platform of device technology.

In what follows we describe our findings. In the Section 2, we demonstrate the Hamiltonian and the basic methodology of generating and engineering the continuous resonant band in a quasiperiodic mesh. Section 3 deals with the evaluation of density of states profile and the subsequent relevant discussion regarding the controlled band tuning. Then in Section 4, the technique of transport calculations is presented to justify our analytical treatment. Finally, we draw our conclusions. 


\section{MODEL AND HAMILTONIAN}

First we will start our discussion with the prototype geometry comprising of two-channel copper-mean ladder network as cited in the Fig. 1. Ordinary copper-mean chain has two types hopping integral parameters, in the tight-binding description, viz., 'long' $(A)$ and 'short' $(B)$ "bonds and the network grows following the chronological sequence: $A \rightarrow A B B, B \rightarrow A$. Accordingly, the successive generations are $G_{1}=A, G_{2}=A B B, G_{3}=A B B A A$, $G_{4}=A B B A A A B B A B B$ and so on. Following this quasi-periodic sequence one can in principle, periodically couple an enormous number of such infinitely extended (along $X$-axis) copper-mean arrays in the transverse direction or even without periodicity. We discuss here the $N$-legged copper-mean mesh which has periodicity along transverse axis.

Any type of this multi-strand quasi-periodic ladder systems can be described in the standard tight-binding formalism by the following Hamiltonian in the Wannier basis for the spinless non-interacting electrons, viz.,

$$
H=\sum_{n, k} \epsilon_{n}^{k} c_{n}{ }^{k} c_{n}{ }^{k}+\sum_{\langle n m\rangle, k} t_{n m}^{k} c_{n}^{k^{\dagger}} c_{m}{ }^{k}+\sum_{n,\langle k l\rangle} \Gamma_{n} c_{n}{ }^{k^{\dagger}} c_{n}{ }^{l}+\sum_{\langle n m\rangle, k} \chi_{n m}^{k, k+1} c_{n}{ }^{k^{\dagger}} c_{m}{ }^{k+1}+\sum_{\langle n m\rangle, k} \chi_{n m}{ }^{k, k-1} c_{n}{ }^{k^{\dagger}} c_{m}{ }^{k-1}
$$

The first part of the Hamiltonian signifies the potential contribution while the other terms carry the kinetic contribution. The pairs of indices $(n, m)$ are associated with nearest neighbor atomic sites on any particular strand, while $k$ and $l$ index represent different strands in the mesh. We can distinguish between the different types of quantum dots present in the system. There are basically four kinds of atomic sites in each strand depending on the local environment, which are $\alpha$ (green colored spheres) sites, $\beta$ (red colored spheres) sites, $\gamma$ (purple colored spheres) sites and $\delta$ (cyan colored spheres) kind of sites respectively flanked by pairs of bonds $A-A, A-B$, $B-A$ and $B-B$. The on-site potentials of these kind of sites are designated as $\epsilon_{\alpha}, \epsilon_{\beta}, \epsilon_{\gamma}$ and $\epsilon_{\delta}$ respectively. The nearest neighbor hopping integrals in any strand are assigned values $t_{A}, t_{B}$, across the $A$ and the $B$ bonds respectively. We additionally introduce second neighbor connection between pairs of strands across the diagonals in the bigger and the smaller rectangular plaquettes as shown, and denote them by $\chi_{n m}{ }^{k, k \pm 1}=\chi_{A}$ or $\chi_{B}$ respectively according to the geometry. The inter-strand tunnel hopping, connecting the $i$-th site in the $k$-th strand with the $n$-th site in the $l$-th strand $(l=k \pm 1)$ is $\Gamma_{n}=\Gamma_{\alpha}$, $\Gamma_{\beta}, \Gamma_{\gamma}$ or $\Gamma_{\delta}$ depending on whether it connects $\alpha, \beta$, $\gamma$ or $\delta$ sites of the neighboring strands along the transverse axis. The provision of tuning the inter-strand hopping parameters or the second neighbor hopping integrals makes the model more realistic implying that one can, in principle, talk about the quasiperiodically distorted ladder networks or double stranded DNA-like models [31, 32] as well. This definitely brings a flavor of geometrical disorder and hence we can discuss its effect on the spectral landscape of such systems within the same formalism. Also, second motivation of this work is to study the tunable thermo-electric transport in the next part as a result of such quasiperiodically modulated geometry.

By virtue of the generalized difference equation (which is the discretized version of the Schrödinger's equation) given by

$$
\left(E-\epsilon_{n}\right) \psi_{n}=\sum_{m} t_{n m} \psi_{m}
$$

we can easily obtain, for any such $N$-strand quasiperiodic mesh, $3 N$ difference equations. Therefore, for coppermean ladder, there are $N$-equations corresponding to each vertical rung with $\alpha, \beta, \gamma$ or $\delta$ sites residing on it. To avoid complicacy regarding generalized form of such difference equations, we focus on a two-strand only. It is needles to say that this formalism can be extrapolated for any $N$-legged quasi-periodic ladder. Moreover, this is enough to bring out the central spirit of the calculations, and a generalization to the case of arbitrary $N$ is quite trivial.

For a two-strand network, the difference equations for an $\alpha$-rung read,

$$
\begin{aligned}
\left(E-\epsilon_{\alpha}\right) \psi_{i, 2} & =t_{L} \psi_{i+1,2}+t_{L} \psi_{i-1,2} \chi_{L} \psi_{i+1,1}+\chi_{L} \psi_{i-1,1}+\Gamma_{\alpha} \psi_{i, 1} \\
\left(E-\epsilon_{\alpha}\right) \psi_{i, 1} & =t_{L} \psi_{i+1,1}+t_{L} \psi_{i-1,1}+\chi_{L} \psi_{i+1,2}+\chi_{L} \psi_{i-1,2}+\Gamma_{\alpha} \psi_{i, 2}
\end{aligned}
$$


responding sites. All these difference equations corresponding to each rung can be easily recast in a combined matrix form viz.,

$$
(E I-\epsilon) \psi_{n}=t_{n+1} \psi_{n+1}+t_{n-1} \psi_{n-1}
$$

Here,

$$
\begin{gathered}
\boldsymbol{I}=\left(\begin{array}{ll}
1 & 0 \\
0 & 1
\end{array}\right) \\
\boldsymbol{\epsilon}=\left(\begin{array}{ll}
\epsilon_{n, 2} & \Gamma_{21} \\
\Gamma_{12} & \epsilon_{n, 1}
\end{array}\right) \\
\boldsymbol{\psi}_{\boldsymbol{n}}=\left(\begin{array}{l}
\psi_{n, 2} \\
\psi_{n, 1}
\end{array}\right) \\
\boldsymbol{t}_{\boldsymbol{n} \pm \mathbf{1}}=\left(\begin{array}{c}
t_{i, i \pm 1}^{2} \\
\chi_{i, i \pm 1}^{12} \\
t_{i, i \pm 1}^{121}
\end{array}\right) \\
\boldsymbol{\psi}_{\boldsymbol{n} \pm \mathbf{1}}=\left(\begin{array}{l}
\psi_{n \pm 1,2} \\
\psi_{n \pm 1,1}
\end{array}\right)
\end{gathered}
$$

It is to be noted that the 'potential matrix' (comprising of the on-site potential and the inter-strand coupling) and the 'hopping matrix' (containing nearest neighbor and second neighbor connections) commute with each other, and hence can be simultaneously diagonalized by a similarity transform. Eq. (4) can then be easily decoupled, in a new basis defined by the following relation

$$
\left(\begin{array}{c}
\phi_{2} \\
\phi_{1}
\end{array}\right)=\boldsymbol{M}^{-1}\left(\begin{array}{l}
\psi_{2} \\
\psi_{1}
\end{array}\right)
$$

The matrix $\boldsymbol{M}$ diagonalizes both the 'potential' and the 'hopping' matrices. We can make therefore a uniform change of basis and in the new basis, we are left with four independent linear difference equations for each of the rungs. Each of these equations represents a coppermean chain describing a kind of pseudoparticles with states that are linear combinations of the old Wannier orbitals. The decoupled, independent, linear equations are:

$$
\begin{aligned}
{\left[E-\left(\epsilon_{\alpha}-\Gamma_{\alpha}\right)\right] \phi_{i, 2}=\left(t_{L}-\chi_{L}\right) \phi_{i+1,2}+} \\
\left(t_{L}-\chi_{L}\right) \phi_{i-1,2} \\
{\left[E-\left(\epsilon_{\beta}-\Gamma_{\beta}\right)\right] \phi_{i, 2}=\left(\begin{array}{c}
\left.t_{S}-\chi_{S}\right) \phi_{i+1,2}+ \\
\left(t_{L}-\chi_{L}\right) \phi_{i-1,2}
\end{array}\right.} \\
{\left[E-\left(\epsilon_{\gamma}-\Gamma_{\gamma}\right)\right] \phi_{i, 2}=\left(\begin{array}{c}
\left.t_{L}-\chi_{L}\right) \phi_{i+1,2}+ \\
\left(t_{S}-\chi_{S}\right) \phi_{i-1,2}
\end{array}\right.} \\
{\left[E-\left(\epsilon_{\delta}-\Gamma_{\delta}\right)\right] \phi_{i, 2}=\left(\begin{array}{c}
\left.t_{S}-\chi_{S}\right) \phi_{i+1,2}+ \\
\left(t_{S}-\chi_{S}\right) \phi_{i-1,2}
\end{array}\right.}
\end{aligned}
$$

$$
\begin{gathered}
{\left[E-\left(\epsilon_{\alpha}+\Gamma_{\alpha}\right)\right] \phi_{i, 1}=\left(t_{L}+\chi_{L}\right) \phi_{i+1,1}+} \\
\left(t_{L}+\chi_{L}\right) \phi_{i-1,1} \\
{\left[E-\left(\epsilon_{\beta}+\Gamma_{\beta}\right)\right] \phi_{i, 1}=\left(\begin{array}{c}
\left.t_{S}+\chi_{S}\right) \phi_{i+1,1}+ \\
\left(t_{L}+\chi_{L}\right) \phi_{i-1,1}
\end{array}\right.} \\
{\left[E-\left(\epsilon_{\gamma}+\Gamma_{\gamma}\right)\right] \phi_{i, 1}=\left(\begin{array}{c}
\left.t_{L}+\chi_{L}\right) \phi_{i+1,1}+ \\
\left(t_{S}+\chi_{S}\right) \phi_{i-1,1}
\end{array}\right.} \\
{\left[E-\left(\epsilon_{\delta}+\Gamma_{\delta}\right)\right] \phi_{i, 1}=\left(\begin{array}{c}
\left.t_{S}+\chi_{S}\right) \phi_{i+1,1}+ \\
\left(t_{S}+\chi_{S}\right) \phi_{i-1,1}
\end{array}\right.}
\end{gathered}
$$

Each of the equations Eq. (11) - Eq. (12) yields two independent copper-mean chains with the corresponding effective on-site potentials $\left[\left(\epsilon_{\alpha} \pm \Gamma_{\alpha}\right),\left(\epsilon_{\beta} \pm \Gamma_{\beta}\right),\left(\epsilon_{\gamma} \pm \Gamma_{\gamma}\right)\right.$, $\left.\left(\epsilon_{\delta} \pm \Gamma_{\delta}\right)\right]$. Also, the effective hopping integral parameters are $t_{L} \pm \chi_{L}, t_{S} \pm \chi_{S}$. Further note that when we consider individual set of the equations, each set gives rise to typical spectrum of copper mean chain containing a broad continua populated by extended eigenfunctions (or eigenfunctions with large localization lengths). Thus, it is needless to say that the actual spectra of the CMladder network is just the convolution of the individual spectrum. Now we should make comment on the nature of the eigenstates for this multi-legged CM ladder network before ending this section. This can be made possible if we look at the decoupled equations. From the basis transformation formalism, one can easily say that the wavefunctions $\phi_{i}$ 's in the changed basis are the linear superposition of the original amplitudes $\psi_{i}$ 's. Therefore, localization character of any $\phi_{i}$ will prevail only if all the contributing eigenfunctions carry the same character. On the contrary, if by means of some correlations between the parameters, one can make any of the "channel" transparent to the incoming wave-train, then it will render the entire linear combination $\phi_{i}=\sum_{j} \psi_{j}$ conducting.

\section{A. Spectral Information by RSRG method}

To obtain the spectral landscape of this multi-strand quasi-periodic ladder model, we use the standard real space renormalization group method using the potential matrix and hopping matrix respectively given by the Eq. (6) and Eq. (8). In this scheme, we decimate out an appropriate subset of the vertices in terms of the amplitudes of the surviving nodes. Thus for the CM ladder, the decimation procedure is implemented via the backward folding, viz., $A B B \rightarrow A^{\prime}$ and $A \rightarrow B^{\prime}$. It is simple to work out the RSRG recursion relations containing the renormalized potential and hopping and are given by, 


$$
\begin{aligned}
\boldsymbol{\epsilon}_{\alpha, n+1} & =\boldsymbol{\epsilon}_{\gamma, n}+\boldsymbol{t}_{S, n}^{T}\left(E . \boldsymbol{I}-\boldsymbol{\delta}_{1, n}\right)^{-1} \boldsymbol{t}_{S, n}+\boldsymbol{t}_{L, n}\left(E . \boldsymbol{I}-\boldsymbol{\delta}_{2, n}\right)^{-1} \boldsymbol{t}_{L, n}^{T} \\
\boldsymbol{\epsilon}_{\beta, n+1} & =\boldsymbol{\epsilon}_{\gamma, n}+\boldsymbol{t}_{S, n}^{T}\left(E . \boldsymbol{I}-\boldsymbol{\delta}_{1, n}\right)^{-1} \boldsymbol{t}_{S, n} \\
\boldsymbol{\epsilon}_{\gamma, n+1} & =\boldsymbol{\epsilon}_{\alpha, n}+\boldsymbol{t}_{L, n}\left(E . \boldsymbol{I}-\boldsymbol{\delta}_{2, n}\right)^{-1} \boldsymbol{t}_{L, n}^{T} \\
\boldsymbol{\epsilon}_{\delta, n+1} & =\boldsymbol{\epsilon}_{\alpha, n} \\
\boldsymbol{t}_{L, n+1} & =\boldsymbol{t}_{L, n}\left(E . \boldsymbol{I}-\boldsymbol{\epsilon}_{\beta, n}\right)^{-1} \boldsymbol{t}_{S, n}\left(E . \boldsymbol{I}-\boldsymbol{\delta}_{1, n}\right)^{-1} \boldsymbol{t}_{S, n} \\
\boldsymbol{t}_{S, n+1} & =\boldsymbol{t}_{L, n} \\
\boldsymbol{\delta}_{1, n} & =\boldsymbol{\epsilon}_{\delta, n}+\boldsymbol{t}_{S, n}^{T}\left(E . \boldsymbol{I}-\boldsymbol{\epsilon}_{\beta, n}\right)^{-1} \boldsymbol{t}_{S, n} \\
\boldsymbol{\delta}_{2, n} & =\boldsymbol{\epsilon}_{\beta, n}+\boldsymbol{t}_{S, n}\left(E . \boldsymbol{I}-\boldsymbol{\epsilon}_{\delta, n}\right)^{-1} \boldsymbol{t}_{S, n}^{T}
\end{aligned}
$$

For the numerical evaluation of the density of states, we add a small imaginary part to the energy. Following the above recursion relations the hopping integrals flow to zero after certain number of iterations and potential part will reach to its fixed point value. We then calculate the local green's function $\boldsymbol{G}=\left(E . \boldsymbol{I}-\boldsymbol{\epsilon}_{\boldsymbol{i}}^{*}\right)^{-1}$ where the subscript $i$ may be different corresponding to the different type of nodes. The 'asterix' mark denotes the fixed point value of the on-site term. Finally, the diagonal term of the Green's function matrix will give the local spectral information of any particular site.

\section{BAND ENGINEERING}

The advantage of the decoupling scheme lies in the fact that one can have the liberty to engineer the extent of

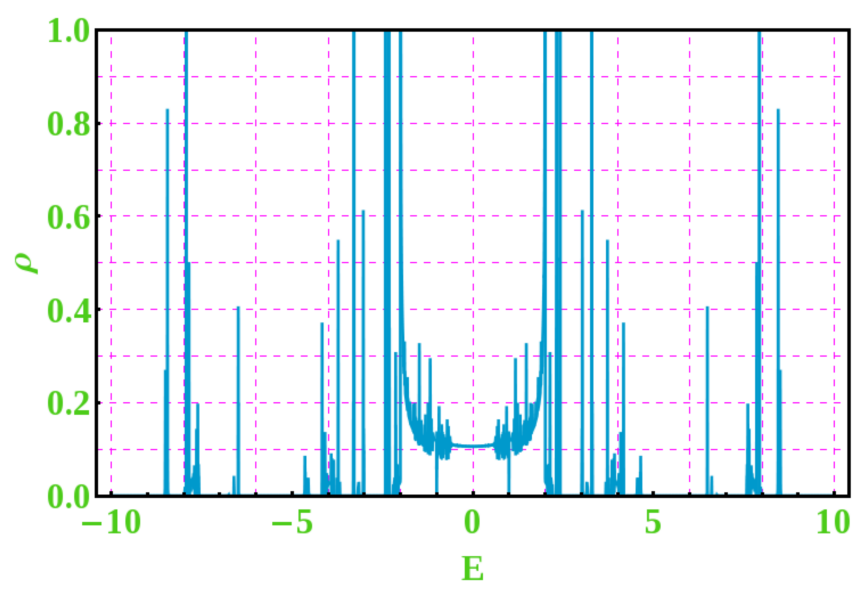

FIG. 2: (Color online) (a) The spectral landscape of Density of States with Energy. We have set $\epsilon_{i}=0, t_{L}=1$ and, $\chi_{L}=2, \chi_{S}=3$. Also, all the vertical couplings $\Gamma_{\alpha}, \Gamma_{\beta}$ and $\Gamma_{\gamma}$ are set equal to $\Gamma$ for numerical calculation.

absolutely continuous energy bands even in this kind of system where there is no question of translational invariance. Such type of correlation does not bother about the individual values of the parameters of the Hamiltonian. So, taking in terms of some realistic DNA kind of model, one can choose the relevant values of the parameters taken from the simulation technique. We justify the above claim by pointing out to the fact that, for example, in equation (11), if we set all $\epsilon_{i}-\Gamma_{i}$ same and equal to constant for all $i$ and $t_{L}-\chi_{L}=t_{S}-\chi_{S}$, then the set of equation (11) represents a perfectly ordered system with the corresponding band edge extending from $E=\left(\epsilon_{\alpha}-\Gamma_{\alpha}\right)-2\left(t_{L}-\chi_{L}\right)$ to $E=\left(\epsilon_{\alpha}-\Gamma_{\alpha}\right)+2\left(t_{L}-\chi_{L}\right)$. Note that this continuous bands will be populated by $e x-$ tended, Bloch-like pseudo-particle eigenstates.

It is to be that the correlation made for band engineering does not impose any restriction on the individual values of the on-site potential $\epsilon_{i}$ and inter-strand connection $\Gamma_{i}$. This only needs that the differences $\epsilon_{i} \pm \Gamma_{i}$ and $t_{i j}^{k}-\chi_{i j}^{k, k \pm 1}$ have to remain unchanged. In an exactly similar fashion, if we look at the other set of decoupled difference equation for this quasiperiodic mesh, parallel observation can be obtained, where the correlations needed for the generation of resonant conducting band are $\left(\epsilon_{\alpha}+\Gamma_{\alpha}\right)=\left(\epsilon_{\beta}+\Gamma_{\beta}\right)=\left(\epsilon_{\gamma}+\Gamma_{\gamma}\right)=\left(\epsilon_{\delta}+\Gamma_{\delta}\right)$ and $t_{L}+\chi_{L}=t_{S}+\chi_{S}$.

In Fig. 2, we show the density of states profile of a two strand copper-mean ladder with all on-site energy $\epsilon_{i}=0$ and following the correlations mentioned above. Here, we have set, without any loss of generality, $\Gamma_{\alpha}=\Gamma_{\beta}=\Gamma_{\gamma}=\Gamma_{\delta}=0$, just to make the center of the spectrum at $E=0$, and the inter-strand connection happens through the diagonal hopping integral term only. Now it is to be mentioned that any one of those two decoupled difference equations will at a time contribute to generate an absolutely continuous band populated by extended Bloch-like states only. The other equation will then populate the spectrum with critical eigenstates, typical characteristics of the quasiperiodic arrangement. The convolution of those two different eigenstates obtained from two decoupled equations under parametric correlation will demonstrate the complete spectral information. Therefore, if some of the critical states happen to be occupying part of the spectrum that falls within the central continuous zone, then they will loss their critical identity and become a member of extended family. This is actually what happens in the Fig. 2. Here we incorporate the correlation between the 
nearest neighbor hopping integrals and next nearest connection as $t_{L}+\chi_{L}=t_{S}+\chi_{S}$ with $t_{L}=1, t_{S}=2$, $\chi_{L}=2, \chi_{S}=3$. As soon as we set the correlation, one difference equation represents a perfectly periodic chain of identical atomic sites that contributes an absolutely continuous band populated by extended eigenfunctions having extent between $-2 \leq E \leq 2$. The other difference equation will obviously give the spectrum that carries the flavor of quasiperiodic copper-mean geometry. The full spectrum, as obtained from RSRG recursion relation and the Green's functions reproduce the absolutely continuum exactly over the energy regime, as extracted from decoupled equation. (Eq. (11)).

The resonant character of those states belonging to the central continuum can be easily justified following the usual method of checking the flow of hopping integrals under successive RSRG iterations. We have carefully scanned the continuous zone over arbitrary small energy intervals. For any arbitrary energy picked up from the continuum, the hopping integral retain oscillatory nature without showing any signature of convergence to zero for an arbitrary large number of loops. This brings a clear indication that the corresponding Wannier orbitals have finite non-zero overlap over arbitrarily large distances basic characteristic feature of the extended eigenfunction.

In a similar manner it is very easy to perform the same checking of the flow of hopping parameter under successive iteration steps for the single particle states situated at the flank of the eigenspectrum. The flow will converge to zero after a moderate number (small compared to that of central transparent states) of RSRG steps. This immediately suggests that the corresponding eigenfunctions at least have very large localization lengths (if not considered to be extended). At this point we should mention that we have used the above decoupled equations only to extract the region of the central (in this case) continuous subband. The profile of density of eigenstates presented here is obtained by using the RSRG method on the full two-strand quasiperiodic mesh.

\section{TRANSPORT CHARACTERISTICS}

To corroborate our findings we will now do parametric calculation to elucidate the transport characteristics of the quasiperiodic coppermean ladder like systems following the usual method of multichannel transport. For this purpose, we need to focus our attention on the systems where a finite size two strand coppermean ladder network is connected with a pair of semi-infinite leads as shown in the Fig. 3. To evaluate the transmittance, here we now adopt the Green's function approach. Hence we need to define the Green's function for the composite systems (lead-system-lead) which is given by,

$$
G=(E-H)^{-1}
$$

where $E=\epsilon+i \eta$ with $\eta$ being the arbitrarily small number which can be set as zero in the limiting approxi-

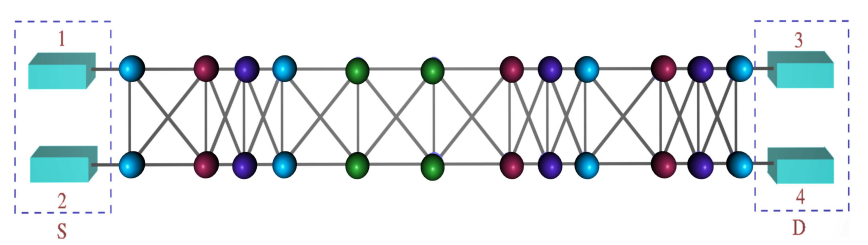

FIG. 3: (Color online) A finite size coppermean ladder network is sandwiched between semi-infinite leads. The different quantum dots are designated by different colors such as green $\operatorname{dot}(\alpha$ site $)$, red $\operatorname{dot}(\beta$ site $)$, purple dot $(\gamma$ site $)$ and cyan dot $(\delta$ site) respectively. Two types of bonds 'long' and 'short' bonds are assigned with two different hopping integrals $t_{A}$ and $t_{B}$ respectively.

mation. $\epsilon$ is the energy of the incoming electron. $\mathrm{H}$ is the Hamiltonian of the entire system including the semi-infinite leads also. So the above Green's function equation deals with the inversion of the matrix of infinite dimension that corresponds to a system consisting of the finite size quasiperiodic mesh and semi-infinite leads. Therefore, if we write the Hamiltonian explicitly for the individual subsystem then we have,

$$
H=H_{M}+\sum_{m=1}^{N}\left(H_{m}+H_{m M}+H_{m M}^{\dagger}\right)
$$

where $H_{M}$ and $H_{m}$ are the Hamiltonians respectively for the original finite size ladder geometry and lead-m-N corresponds to the number of leads to which the system gets connected. $H_{m M}$ denotes the coupling matrix. Further note that all the leads designated with the respective tight-binding parameters have equal footing. also within the tight-binding framework the system is demonstrated by the above Hamiltonian as written in Eq. (15). Following the partition of the Hamiltonian, we can easily write the effective Green's function for the system introducing the Lowdin's partitioning technique $[33,34]$ as,

$$
G_{M}=\left(E-H_{M}-\sum_{m=1}^{N} \Sigma_{m}\right)^{-1}
$$

where $\Sigma_{m}$ offers the self-energy contribution because of the presence of the coupling of the system to the lead. The explicit expression for the self energy corresponding to particular $m^{\text {th }}$ lead is given by,

$$
\Sigma_{m}=H_{m M}^{\dagger} G_{m} H_{m M}
$$

Here $G_{m}=\left(E-H_{m}\right)^{-1}$ is the Green's function of $m^{t h}$ lead. It is needless to mention that the self energy term carries the signature of the entire information regarding coupling between the system and lead. Using the Eq. (17), the coupling function $\Gamma_{m}$ can be easily obtained from the following equation $[35,36]$,

$$
\Gamma_{m}(E)=i\left[\Sigma_{m}^{r}(E)-\Sigma_{m}^{a}(E)\right]
$$


where $\Sigma_{m}^{a(r)}(E)$ signifies the advanced (retarded) self energy terms respectively. It is obvious that they are Hermitian conjugate to each other. Thereafter, we may rewrite the above Eq. (18) as,

$$
\Gamma_{m}=-2 \operatorname{Im}\left(\Sigma_{m}^{r}\right)
$$

Now the transmission co-efficient can be expressed in terms of the effective system and system-to-lead coupling as,

$$
T_{m n}=\operatorname{Tr}\left[\Gamma_{m} G_{M}^{r} \Gamma_{n} G_{M}^{a}\right]
$$

Here $\Gamma_{m}$ and $\Gamma_{n}$ denote the connection of the system to the $m^{\text {th }}$ and $n^{\text {th }}$ lead respectively and $G-M^{r}$ and $G_{M}^{a}$ are the retarded and advanced Green's function of the system respectively. As the coupling matrix $H_{m M}$ is non-zero only for the adjacent points, the expression for the transmission probability becomes [37]

$$
T_{m n}=4 \Delta_{m} \Delta_{n}\left|G_{M}\right|^{2}
$$

The transmission characteristics for this two-strand copper mean ladder network is represented in the Fig. 4

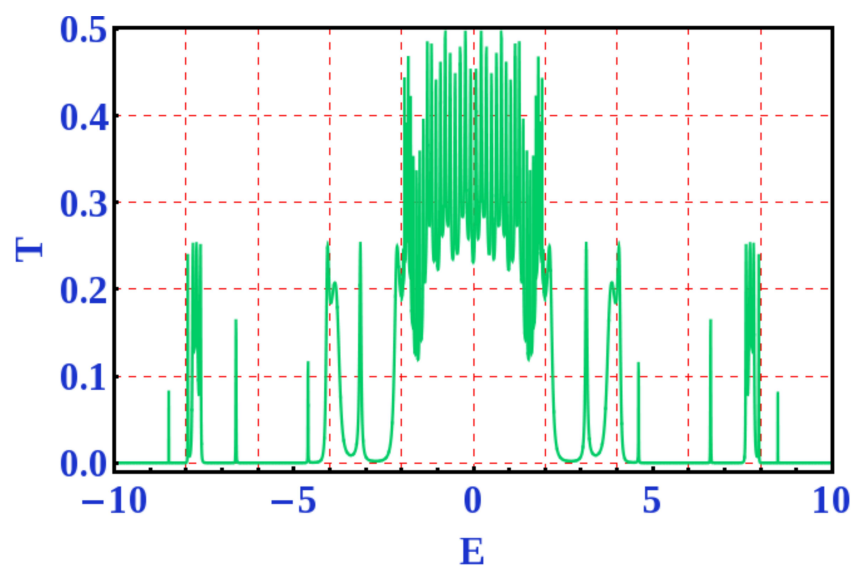

FIG. 4: (Color online) (a) The transmission characteristics of a coppermean ladder with 43 bonds in each arm as a function of energy under the specific correlation. We have set $\epsilon_{i}=0$, $t_{L}=1$ and, $\chi_{L}=2, \chi_{S}=3$. Also, all the vertical couplings $\Gamma_{\alpha}, \Gamma_{\beta}$ and $\Gamma_{\gamma}$ are set equal to $\Gamma$ for numerical calculation.

using the above analytical expression 21. From the graphical plot, it is clear that the system shows resonant behaviour to the incoming electron for the central regime of energy $(-2 \leq E \leq 2)$ where it shows a moderately high transport. Outside this central conducting zone, there exists few discrete set of energies at the flank of the spectrum for which it is showing relatively low transport. The conducting nature of the system basically reflects the signal of quasiperiodic geometry present. The central resonating region is happening due to the arrangements of 'short' bonds.

\section{THERMOELECTRIC BEHAVIOR OF COPPER MEAN LADDER}

We now study the thermoelectric property of this twoarm quasiperiodic ladder geometry. The quantitative measure of thermoelectric transport is manifested by the Seebeck coefficient which solely depends on the transmission characteristics of the system under study showing the signature of the eigenspectrum. Experimental measurement of the Seebeck coefficient [38-41] has been conducted at zero bias. In that case, the system can be described by only a single Fermi level and the Seebeck coefficient can be expressed in terms of $T(E)$ by means of the expression,

$$
S=-\frac{\pi^{2}}{3} \frac{k_{B}^{2} T}{|e|}\left(\frac{\partial \ln T(E)}{\partial E}\right)_{E_{F}}
$$

where $|e|$ is the charge of the electron, $k_{B}$ is the Boltzmann's constant and $T$ is the mean temperature of the contacts. The above expression can be rewritten in terms of Lorentz number as,

$$
S=-|e| L_{0} T\left(\frac{\partial \ln T(E)}{\partial E}\right)_{E_{F}}
$$

where $L_{0}=\frac{\pi^{2}}{3}\left(\frac{k_{B}}{|e|}\right)^{2}$ is called Lorentz number.

In our case, the conductivity mechanism is described following the usual method of multichannel trans-

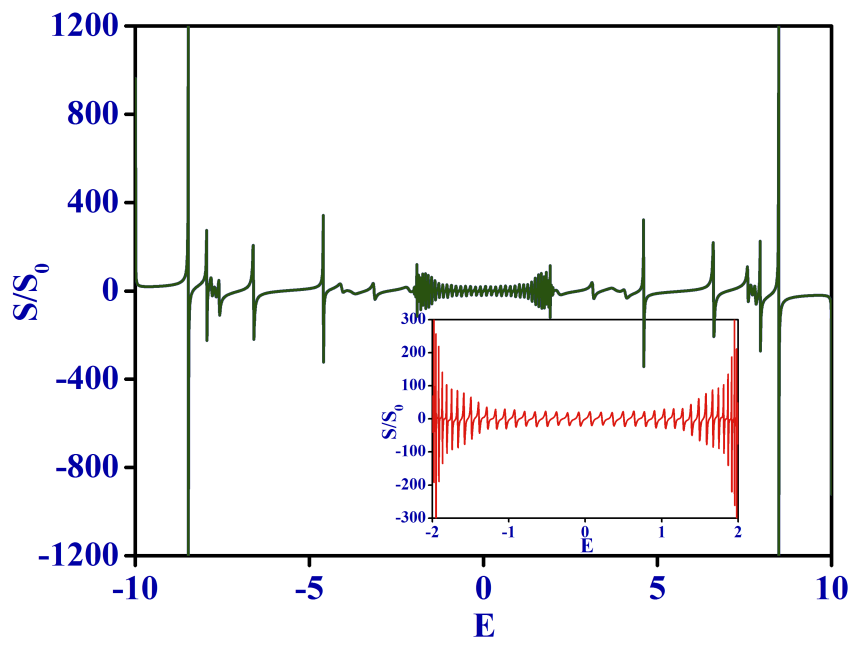

FIG. 5: (Color online) Plot of thermoelectric Seebeck coefficient against energy (E). The term $S_{0}$ contains all the terms of Eq. (23) excluding the derivative part. If the hopping integrals are taken in unit of $e V$ then $S$ has the unit of $\mu V K^{-1}$.

port [42-45] (as mentioned earlier). Then by using the above expression for Seebeck coefficient we have obtained the behavior of thermoelectric transport of the quasiperiodic ladder network. Fig. 5 shows the variation of thermoelectric Seebeck coefficient of a finite size two-strand ladder arranged in a quasiperiodic coppermean geometry, sandwiched between pairs of semi-infinite ordered leads 
against the energy $E$ of the incoming electron. The plot shows unusual behavior due to the presence of the minigaps in the electronic spectrum. From the figure it is clear that the magnitude of the Seebeck coefficient diminishes slightly as we move from the outer regime to the central continuum part of energy spectrum. Interestingly, however, besides small fluctuations near the subband edges, the Seebeck coefficient shows strong oscillations near the minigaps. Moreover, throughout the whole range of energy it shows several sharp transition between positive and negative magnitude mimicking a Fano-like nature. This repetitive sign change in the Seebeck coefficient as the Fermi energy varies has significant implications, indicating that the system may be tailored to exhibit n-or ptype properties using the same dopant (electron donors) by carefully controlling the range of energy and also by selective choice of the tight-binding parameters of the network. It is to be noted that a similar behavior of enhanced Seebeck coefficient due to miniband formation has also been reported by Balandin et. al. [46] for three dimensional quantum dot array structures.

It is important to mention emphatically that the precise numerical correlation between the parameters of the Hamiltonian can give a boost to engineer the band spectrum at will. This immediately affects the transport and hence the thermoelectric behavior. So with the aid of band tuning one can easily control the thermoelectric transport in a subtle way by virtue of parametric correlations. This observation is quite significant as this illustrates the advantage of tunable thermoelectricity of such aperiodic geometry which could be convenient in the thermoelectric application oriented aspects.

\section{CLOSING REMARKS}

We have addressed the problem of observing transparent energy states in a system that is quasi-one dimensional and non-translationally invariant. In the present arena of lithographic and nanotechnology methodologies, and in particular, with the rapid advancement in the controlled growth of optical lattices with trapped BoseEinstein condensates, such systems can, in principle, be made in practice. This has already achieved the milestone in perceiving the Anderson localization of matter waves, and in the field of optics as well. Our analysis reveals that, one can engineer sub-bands which are absolutely continuous and holds Bloch functions only (even in the absence of translational invariance on a global scale). The salient point of observation is that, for this one needs to correlate the numerical values of a subset of the system parameters in a special way. This observation brings forth a highly non-trivial variation of Anderson localization that can be monitored, and observed in low dimensional systems. The results are sound, specially when academic people are going for direct experimental measurement of localization related properties in low dimensional lattices, and hence may trigger further experiments on grafted lattices in low dimensions.

Specifically, here we have taken the example of copper mean sequence. In this work we have computed the density of states (DOS) and transport using a real space renormalization group method (RSRG). A commutation relation between the potential and hopping matrix has been exploited to work out the definite correlations between the parameters that can form absolutely continuous bands. The numerical correlations attained from the commutation play a pivotal role in the crossover of electronic eigen spectrum from localization to completely continuum bands. This scenario obviously adds up a flavor of non-triviality in the variation of the canonical case of Anderson localization. Finally, the tunable thermoelectric transport has been formulated for this quasiperiodic two-strand mesh.

\section{Acknowledgments}

A.M. gratefully acknowledges the financial support through an INSPIRE fellowship [IF160437] from DST, India. A.N. is thankful to the Department of Physics, University of Kalyani, W. B., India for providing the computational facility. Both the authors are grateful for the stimulating discussions regarding the results with Prof. Arunava Chakrabarti.
[1] P. W. Anderson, Phys. Rev. 109, 1492 (1958).

[2] B. Kramer and A. MacKinnon, Rep. Prog. Phys. 56, 1469 (1993).

[3] E. Abrahams, P. W. Anderson, D. C. Licciardello, and T. V. Ramakrishnan, Phys. Rev. Lett. 42, 673 (1979).

[4] H. Hu, A. Strybulevych, J. H. Page, S. E. Skipetrov, and B. A. Van Tiggelen, Nat. Phys. 4, 945 (2008).

[5] J. Billy, V. Josse, Z. Zuo, A. Bernard, B. Hambrecht, P. Lugan, D. Clèment, L. S.- Palencia, P. Bouyer, and A. Aspect, Nat. (London) 453, 891 (2008).

[6] A. Christ, Y. Ekinci, H. H. Solak, N. A. Gippius, S. G. Tikhodeev, and O. J. F. Martin, Phys. Rev. B 76,
201405(R) (2007).

[7] F. Rüting, Phys. Rev. B 83, 115447 (2011).

[8] J. O. Vasseur, P. A. Deymier, G. Frantziskonis, G. Hong, B. Djafari-Rouhani, and L. Dobrzynski, J. Phys.: Condens. Matter 10, 6051 (1998).

[9] I. O. Barinov, A. P. Alodzhants, and S. M. Arakelyan, Quantum Electron. 39, 685 (2009).

[10] S. Mukherjee, A. Spracklen, D. Choudhury, N. Goldman, P. Öhberg, E. Andersson, and R. R. Thomson, Phys. Rev. Lett. 114, 245504 (2015).

[11] S. Mukherjee and R. R. Thomson, Opt. Lett. 40, 5443 (2015). 
[12] Y. Gilead, M. Verbin, and Y. Silberberg, Phys. Rev. Lett. 115, 133602 (2015).

[13] J. Svozilík, J. Peřina, Jr., and J. P. Torres, Phys. Rev. A 89, 053808 (2014).

[14] D. H. Dunlap, H-L. Wu, and P. W. Phillips, Phys. Rev. Lett. 65, 88 (1990).

[15] W. Zhang and S. E. Ulloa, Phys. Rev. B 69, 153203 (2004).

[16] S. Sil, S. K. Maiti, and A. Chakrabarti, Phys. Rev. B 78, 113103 (2008).

[17] A. Rodriguez, A. Chakrabarti, and R. A. Römer, Phys. Rev. B 86, 085119 (2012).

[18] B. Pal, S. K. Maiti, and A. Chakrabarti, Europhys. Lett. 102, 17004 (2013).

[19] B. Pal and A. Chakrabarti, Eur. Phys. J. B 85, 307 (2012).

[20] A. Chakrabarti, S. N. Karmakar, and R. K. Moitra, Phys. Rev. B 39, 9730 (1989).

[21] A. Nandy, B. Pal, and A. Chakrabarti, Europhys. Lett. 115, 37004 (2016).

[22] S. Sil, S. N. Karmakar, R. K. Moitra, and A. Chakrabarti, Phys. Rev. B 48, 4192(R) (1993).

[23] A. Mukherjee, A. Nandy, and A. Chakrabarti, Eur. Phys. J. B 90, 52 (2017).

[24] A. Aviram and M. A. Ratner, Chem. Phys. Lett. 29, 277 (1974).

[25] W. U. Huynh, J. J. Dittmer, and A. P. Alivisatos, Science 295, 2425 (2002).

[26] G. Yu, J. Gao, J. C. Hummlen, F. Wudl, and A. J. Heeger, Science 270, 1789 (1995).

[27] J. C. Poler, R. M. Zimmermann, and E. C. Cox, Langmuir 11, 2689 (1995).

[28] E. Maciá, Phys. Rev. B 75, 035130 (2007).

[29] M. Paulsson and S. Datta, Phys. Rev. B 67, 241403(R)
(2003).

[30] X. Gao, K. Uehara, D. D. Klug, J. S. Tse, and T. M. Tritt, Phys. Rev. B 72, 125202 (2005).

[31] S. Kundu and S. N. Karmakar, Phys. Rev. B 89, 032719 (2014).

[32] S. Kundu and S. N. Karmakar, Phys. Lett. A 380, 2395 (2016).

[33] P.O. Lowdin, J. Mol. Spectrosc. 10, 12 (1963).

[34] P.O. Lowdin, J. Math. Phys. 3, 969 (1962).

[35] S. Datta, Electronic Transport in Mesoscopic Systems, Cambridge University Press, Cambridge, 1997.

[36] S. Datta, Quantum Transport: Atom to Transistor, Cambridge University Press, Cambridge, 2005.

[37] V. Mujica, M. Kemp, M.A. Ratner, J. Chem. Phys. 101 6849 (1994).

[38] E. Maciá, Phys. Rev. B 82, 045431 (2010).

[39] L. A. Zotti, M. Bürkle, F. Pauly, W. Lee, K. Kim, W. Jeong, Y. Asai, P. Reddy, and J. C. Cuevas, New J. Phys. 16, 015004 (2014).

[40] A. M. Lunde and K. Flensberg, J. Phys.: Condens. Matt. 17, 3879 (2005).

[41] Y. Dubi and M. Di Ventra, Rev. Mod. Phys. 83, 131 (2011).

[42] P. Dutta, S. K. Maiti, and S. N. Karmakar, AIP Adv. 4, 097126 (2014).

[43] P. Dutta, S. K. Maiti, and S. N. Karmakar, Organic Elect. 11, 1120 (2010).

[44] P. Dutta, S. K. Maiti, and S. N. Karmakar, J. Appl. Phys. 114, 034306 (2013).

[45] S. K. Maiti, Solid State Comm. 150, 1269 (2010).

[46] A. A. Balandin and O. L. Lazarenkova, Appl. Phys. Lett. 82, 415 (2003). 